\title{
Sostenibilidad de Bancos Comunitarios de Semillas Criollas y Acriollas en el norte de Nicaragua
}

\author{
Leonel Aarón Vílchez Ponce ${ }^{1}$ \\ Jonny Antonio González Benavidez² \\ Eveling Giomar Lanuza Morales ${ }^{3}$ \\ Oscar Rafael Lanuza Lanuza ${ }^{4}$
}

\section{RESUMEN}

La presente investigación es untrabajo monográfico para optar al titulo de Lic. En Ciencias Ambientales. Esta investigación se realizo dentro del convenio de la FAREM Estelí y la UNAG.

En el artículo se encontrará información relacionada sobre el contexto geográfico de los departamentos estudiados, objetivos, metodología utilizada, caracterización de los Bancos, nivel de sostenibilidad y alternativas de solución.

La metodologia aplicada en esta investigacion, fue mediante fases de estudio: Fase 1: Revision documental, fase 2: Definicion de la muestra, fase 3: Recoleccion de la informacion de campo, fase 4: Analisis de la información recolectada.

En la primera etapa se realizo una caracterización general de los bancos comunitarios, para conocer el estado actual.

A continuación se determino el nivel de sostenibilidad de los bancos con la "Metodología para estimar el nivel de desarrollo Sostenible de los territorios rurales". (Sepúlveda Sergio, et al 2005). Para finalizar se formularon alternativas de solución para mejorar el funcionamiento de los bancos comunitarios.

Palabras claves: Sostenibilidad, semillas criollas, Bancos comunitarios.

1 UNAN-Managua/FAREM-Estelí. Correo Electrónico: leonelaron@yahoo.es

2 UNAN-Managua/FAREM-Estelí. Correo Electrónico: mago88@yahoo.es

3 UNAN-Managua/FAREM-Estelí. Correo Electrónico: eveling.lanuza@gmail.com

4 Tutor del presernte artí́culo, UNAN-Managua/FAREM-Estelí. Correo Electrónico: rafa.artesano@gmail.com 


\title{
Sustainability of Community Banks of Acriollas Seed Hoop in northern Nicaragua
}

\author{
Leonel Aarón Vílchez Ponce ${ }^{1}$ \\ Jonny Antonio González Benavidez ${ }^{2}$ \\ Eveling Giomar Lanuza Morales ${ }^{3}$ \\ Oscar Rafael Lanuza Lanuza ${ }^{4}$
}

\begin{abstract}
This research is a thesis for the degree of B.A. in Environmental Sciences. This research was conducted within the convention between FAREM Estelí and UNAG.

In the article, information related to the geographical context of the departments studied will be found, objectives, methodology, characterization of the Banks, level of sustainability and alternative solutions.
\end{abstract}

The methodology applied in this research was carried out through study phases: Phase 1: Document Revision, Phase 2: Definition of the sample, Phase 3: Collection of the information field, Phase 4: Analysis of the data collected.

In the first phase, a general characterization of community banks was conducted to determine the current state. Then, the level of sustainability of the banks was determined with the "Methodology for estimating the level of sustainable development of rural areas" (Sergio Sepulveda, et al 2005). Finally, alternative solutions were developed to improve the performance of community banks.

Keywords: Sustainability, native seeds, Community Banks.

1 UNAN-Managua/FAREM-Estelí. E-mail: leonelaron@yahoo.es

2 UNAN-Managua/FAREM-Estelí. E-mail: mago88@yahoo.es

3 UNAN-Managua/FAREM-Estelí. E-mail: eveling.lanuza@gmail.com

4 Tutor artícle, UNAN-Managua/FAREM-Estelí. E-mail: rafa.artesano@gmail.com 


\section{INTRODUCCIÓN}

Elpropósito de estainvestigación es plantearalternativas de solución que contribuyan al funcionamiento de los bancos comunitarios de semillas criollas y acriolladas. En el año 1999 nace en Nicaragua el PCaC (programa de campesino a campesino) de la UNAG, dirigido a la conservación y manejo de los Recursos Naturales con énfasis en la producción de alimentos y la seguridad alimentaria (PCaC-UNAG, 2011).

En la actualidad las semillas criollas, las cuales son parte esencial de la agrobiodiversidad local, están sometidas por lo menos a tres grandes amenazas: erosión genética, la contaminación transgenética y la privatización genética (Vásquez, 2009).

Entre las opciones para proteger las semillas criollas nicaragüenses de estas amenazas, esta la iniciativa de promoción de los bancos comunitarios de semillas que varias organizaciones campesinas y $\mathrm{ONG}$, entre ellas el PCaC de la Unión Nacional de Agricultores y Ganaderos (UNAG), y a nivel regional la alianza centroamericana de protección a la biodiversidad, promueven desde hace varios años en el país (Vásquez, 2009).

A pesar que el $\mathrm{PCaC}$ ha estado impulsando el establecimiento de BCSC (Banco Comunitario De Semillas Criollas), llevando varios años realizando investigación y otras actividades sobre semillas criollas no se ha realizado investigaciones que evalúen la sostenibilidad de dichos bancos, razón por la cual el PCaC en convenio con la Facultad Regional Multidisciplinaria, FAREM Estelí decidieron realizar investigaciones en 4 Departamentos en el Norte de Nicaragua (Madriz, Nueva Segovia, Estelí, Matagalpa).

El objetivo general de esta investigación es Evaluar la sostenibilidad de los Bancos Comunitarios de Semillas Criollas y Acriolladas impulsados por el $\mathrm{PCaC}$ en el Norte de Nicaragua.

\section{MATERIALES Y MÉTODOS}

El estudio se realizó en la parte norte del territorio de Nicaragua, comprendida por los departamentos de Estelí, Madriz, Nueva Segovia y Matagalpa. Esta área es una zona sub tropical de transición, las temperaturas promedio oscilan entre $22^{\circ}$ y $27^{\circ}$. Su característica principal es la diversidad del relieve, esta región es ecológicamente muy rica y variada.

Los materiales utilizados fueron cámara fotográfica y libreta de campo. Las herramientas para la recolección de la información fueron entrevistas, guías de observación y grupos focales.

Los datos de campo recolectados a través de los diferentes instrumentos se analizaron de acuerdo a los componentes económicas, ecológicas y sociales para proponer estrategias a fin de contribuir la sostenibilidad de los bancos comunitarios de semillas apoyados por el PCaC.

Se creó una base de datos en el programa de EXCEL y en el programa estadístico SPSS 17.0 para realizar análisis de datos.

Tabla 1. Ubicación de bancos comunitarios de semillas criollas en sus departamentos, municipio y comunidad

\begin{tabular}{|c|c|c|}
\hline Departamento & Municipio & Comunidad \\
\hline Estelí & Condega & $\begin{array}{l}\text { Labranza 2, Laguna santa } \\
\text { rosa, Piedras anchas. }\end{array}$ \\
\hline Madriz & San Lucas & $\begin{array}{l}\text { El coyolito, El naranjo, } \\
\text { El volcán, Los ranchos, } \\
\text { los mangos, Rio arriba, la } \\
\text { Manzana. }\end{array}$ \\
\hline Matagalpa & San Ramón & $\begin{array}{l}\text { El horno 2, Pinares, Baila- } \\
\text { dora, Naranjo, Cerro gran-de } \\
\text { ( } 2 \text { bancos }), \text { Monte gran-de. }\end{array}$ \\
\hline Nueva Segovia & Mozonte & $\begin{array}{l}\text { San Antonio, La cruz, El } \\
\text { Zapote }\end{array}$ \\
\hline
\end{tabular}


Construcción de indicadores de sostenibilidad, basados en la metodología propuesta por (Sepúlveda et al., 2005)

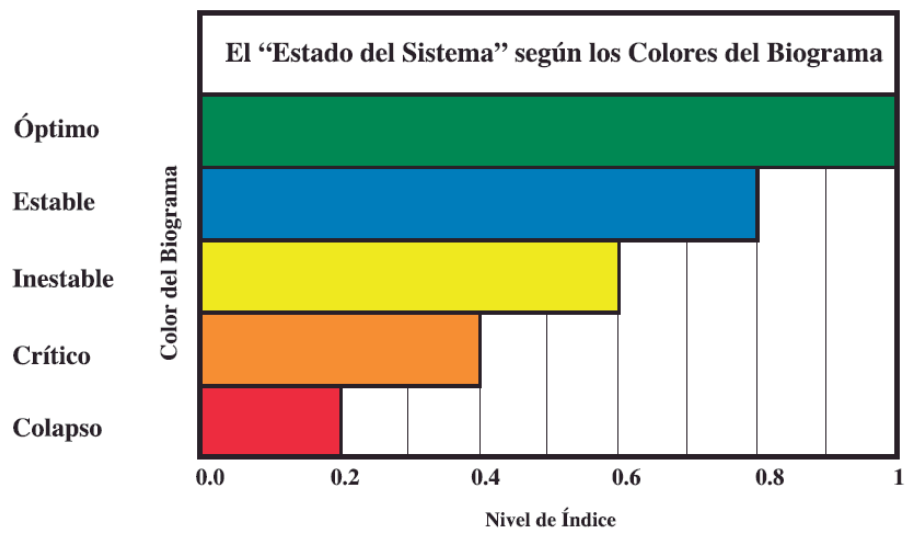

Figura 1. "Metodología para estimar el nivel de Desarrollo Sostenible de los territorios rurales". (Sepúlveda Sergio, Et Al 2005)

El índice integrado de desarrollo sostenible representa la situación general de todo el sistema, y su valor puede variar entre 0 y 1 . Conforme el valor asumido por el índice se va acercando a 1 , el sistema tiene un mejor desempeño de desarrollo. Situación contraria pasa si el índice se va acercando a 0 , ya que el desempeño del sistema va empeorando, facilitando la toma de decisiones futuras que mejoren el funcionamiento de los bancos comunitarios.

Para conocer el índice integrado de sostenibilidad, se aplicaron entrevistas previamente elaboradas, en donde se incluían los componente económico, ecológico y social, a los cuales se les asigno un valor de 100 pts., logrando el valor relativo de cada unidad (banco comunitario de semillas criollas), luego se suman los 3 valores obtenidos, se dividen por el número de componentes, y el valor resultante se divide entre 100 , para así obtener el índice integrado de sostenibilidad.

\section{RESULTADOS Y DISCUSIÓN}

\section{Descripción de los Bancos Comunitarios de Semillas Criollas por departamento.}

\section{Departamento de Matagalpa}

Los bancos comunitario de semillas criollas y acriollas del departamento de Matagalpa fueron fundados por el Programa de Campesino a Campesino ( $\mathrm{PCaC}$ ) entre el 2003 y 2009, desde la fecha de creación, el número de socios fundadores varia de 3 a 20, siendo estos los que aportaron de forma voluntaria su semillas para emprender esta nueva forma de trabajo, favoreciéndose del prestamos de semillas por cada banco un aproximado de 6 productores entre hombres y mujeres.

Debido a este trabajo, se encontró que actualmente estos bancos cuentan con un número de socios que van de un mínimo de 5 y con máximo de 67, favoreciéndose del préstamo de semillas por cada banco un aproximado de 12 productores, entre hombres y mujeres.

La infraestructura de las viviendas está construida con un techo de zinc, piso de tierra, paredes de ladrillo o adobe. La modalidad de funcionamiento que desempeñan los bancos es almacenar semilla comunitaria para prestar.

Las Variedades de maíz y frijoles en los bancos comunitarios de semillas criollas del departamento de Matagalpa son: Maíz: Rosado, Olotillo, Tatacasme, NB6, Rocamel, Amarillo, Tuza blanca, Blanco, H5, Tatacama, Olote rojo. y frijoles son: INTA, Dor, chile, Estelí 90, seda, Nica, palma, $\mathrm{H}$ vaina blanca, $\mathrm{H}$ vaina roja, cuarenteño, CCR, H, Rosa.

Las variedades de maíz con mayor cantidad de semillas en los bancos son: NB6 $(3,454.5 \mathrm{~kg})$, el tatacama $(2,090.9 \mathrm{~kg})$, el Rocamel y Rosa con $454.5 \mathrm{~kg}$ cada uno, en menos cantidades también encontramos Maíz 
H5, Maíz amarillo y olotillo. Entre las variedades de frijoles se encuentran el chile $(2,272.7 \mathrm{~kg})$, Estelí $(4,090.9 \mathrm{~kg})$, DOR $(772.7 \mathrm{~kg})$, el INTA y seda con $454.5 \mathrm{~kg}$ cada uno, en menores sumas hallamos frijol $\mathrm{H}$, $\mathrm{H}$ vaina blanca y frijol Nica.

Los bancos cuentan con los silos y sacos, en algunos casos recipientes plásticos para almacenar semilla, pocos disponen de herramientas para el manejo, como panas, pesas artesanales y zarandas, las que han obtenido con esfuerzo propio, estas en su mayoría se encuentran en buenas condiciones.

Estos bancos se identifican por participar periódicamente en ferias municipales. La mayoría de directivos están capacitados en brindar asistencia técnica en las parcelas de los socios en las situaciones de afectaciones climáticas. Los bancos comunitarios cuentan con libros de registro y reglamento, donde efectúan dos modalidades de préstamo de semillas, una tasa de interés del 100\% para los prestamistas y otra tasa del $50 \%$ para los socios. Una dificultad muy sentida es la entrega de semillas húmedas de productores al banco, debido al clima que predomina en el área.

\section{Departamento de Nueva Segovia}

Los bancos comunitario de semillas criollas y acriollas del departamento de Nueva Segovia fueron fundados por el Programa de Campesino a Campesino ( $\mathrm{PCaC})$ en el año 2003. Estos bancos empezaron a funcionar con número mínimo de socios de 30 un máximo de 70 , donde los mismos productores fueron los beneficiados en ese inicio, por lo que el $\mathrm{PCaC}$ proporcionó la semilla.

Actualmente en los bancos están trabajo un número de socios mínimo de 15 y un máximo de 36 , donde los mismos son los beneficiarios que se favorecen de los servicios del mismo.
Las infraestructuras de las viviendas presentan las condiciones adecuados para el resguardo de las semillas, techadas con zinc, paredes construidas con ladrillo y piso de concreto. La modalidad de funcionamiento del banco es almacenar semilla comunitaria para prestar.

Las Variedades de maíz y frijoles en los bancos comunitarios de semillas criollas del departamento de Nueva Segovia son: Maíz: NB6, Gigante, olote rojo. Y frijol: DOR, INTA, INTA rojo, Rojo de matón 1, Rojo de matón 2, Icalupe, Cuarenteño.

En estas comunidades los bancos cuenta con las herramientas básicas para el manejo, como silos, pesas, zaranda y plástico, encontrándose estas en óptimas condiciones.

La variedad de maíz que albergan mayor cantidad de semillas en los bancos es el NB6 (454.5 kg) luego tenemos el maíz gigante $(136.3 \mathrm{~kg})$ y el olote rojo $(90.9 \mathrm{~kg})$. Con las variedades de frijol encontramos el cuarenteño e INTA rojo con $136.3 \mathrm{~kg}$ cada uno, DOR, Rojo de matón 1, Rojo de matón 2, Icalupe, todos estos con $90.9 \mathrm{~kg}$.

Estas comunidades se identifican por ser pueblos indígenas, debido a estos acostumbran a sembrar variedades adaptadas a la zona, de las que obtienen buenos resultados en las cosechas.

\section{Departamento de Estelí}

Los bancos comunitario de semillas criollas y acriollas del departamento de Estelí fueron fundados por el Programa de Campesino a Campesino ( $\mathrm{PCaC})$ entre los año 2011 y 2003.

Estos bancos presentan las condiciones adecuadas para el resguardo de las semillas, estas bodegas están construidas con techo de zinc, piso de tierra y paredes de adobe especialmente diseñados para realizar esta actividad. La modalidad de funcionamiento de estos 
bancos es almacenar semilla comunitaria para prestar. Las variedades de maíz encontradas tenemos: Olotillo, olote rojo, amarillo, catacama, blanco. Y de frijoles: Zamorano, rojo vaina chata, rojo ceda, maravilla, colombiano, gualiceño, guaqueño, Estelí 90, negro criollo, barreño.

Los bancos de estas comunidades cuenta con las herramientas básicas para el manejo, como: silos, pesas artesanal, zaranda, encontrándose en buenas condiciones, también cuentan con recibos para un mejor control de los prestamistas.

En estas comunidades las variedades de maíz que albergan mayor cantidad de semilla son las siguientes: maíz blanco $(227.27 \mathrm{~kg})$, tatacama y amarillo (159.09 $\mathrm{kg}$ cada uno), de igual manera las variedades de frijol más destacadas son: zamorano $(159.09 \mathrm{~kg})$, negro criollo y barreño (136.2 $\mathrm{kg}$ cada uno).

Estas comunidades se caracterizan por su organización, responsabilidad y entusiasmo de sus directivos de trabajar en función de los bancos comunitarios de semillas criollas

\section{Departamento de Madriz}

Los bancos comunitarios de de semillas criollas en el departamento de Madriz fueron apoyados por el $\mathrm{PCaC}$ entre 2008 y 2011, desde la fecha de creación, el número de socios fundadores varía de 5 a 21, favoreciéndose del préstamo de semillas por cada banco un aproximado 18 productores.

Encontramos actualmente que el número de socios es de 2 a 12, Favoreciéndose del préstamo de semillas por cada banco un aproximado de 14 productores, entre hombres y mujeres.

La infraestructura de las viviendas en rústica, compuesta de techo de zinc, piso de concreto o tierra y paredes de ladrillo y adobe. La modalidad de funcionamiento que desempeñan los bancos es almacenar semilla comunitaria para prestar.

Las variedades de maíz en los bancos comunitarios son: Blanco, NB6, Amarillo, Olotillo. Y frijoles son: Negro, 7 panas, INTA rojo, Maravilla, JM, Rojito Naranjo, Vitamina, Chile rojo, Arbolito, Pueblo Nuevo, Zamorano, Estelí 90, Frijol gandul, Canavalia.

Los bancos solo cuentan con silos, las herramientas que utilizan en su momento son prestadas por socios, debido a que ninguna cuenta con equipamiento, estos se encuentran en buenas condiciones.

Las variedades de maíz que albergan mayor cantidad de semillas en los bancos son: NB6 (636.3 kl) luego tenemos Maíz blanco y Olotillo $(45.4 \mathrm{~kg}$ ) cada uno, también encontramos Maíz amarillo con 34.09 kg libras. Con las variedades de frijol tenemos Chile rojo $(363.6 \mathrm{~kg})$, Vitamina $(340.9 \mathrm{~kg})$, Maravilla, Zamorano, y Estelí 90(227.27kg) cada uno, Frijol pueblo Nuevo $(181.8 \mathrm{~kg})$ y en menores cantidades frijol negro, 7 panas, INTA Rojo, JM, Arbolito $(45.4 \mathrm{~kg})$ cada variedad.

Los bancos del departamento de Madriz, están en un proceso de recuperación, a partir de junio del 2013, la UNAG, con fondos del SWISSAID, están impulsado un proyecto especialmente dirigido a los bancos de semillas comunitarios en el departamento, motivo por el cual se encuentran sin equipamiento para su funcionamiento. 
Clasificación de los bancos comunitarios de semillas criollas y acriolladas, impulsados por el $\mathrm{PCaC}$, según el índice de desarrollo sostenible

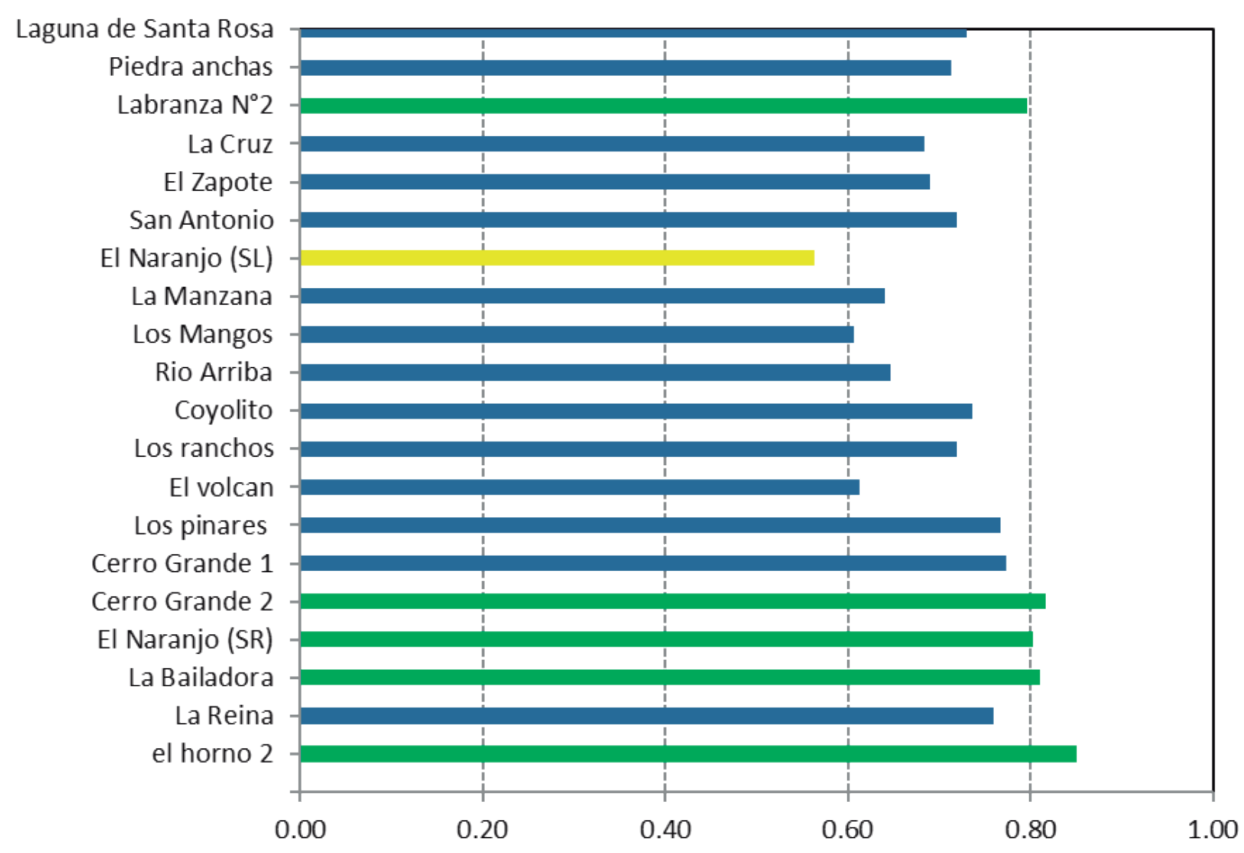

Figura 2. Índice integrado de desarrollo sostenible de los bancos de semillas criollas impulsados por el $\mathrm{PCaC}$, en el norte de Nicaragua.

Estado crítico
Estado de colapso

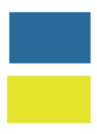

estado estable

estado inestable

En él gráfico se observan evaluados en base aa Metodología utilizada para medir el grado de sostenibilidad (Sepúlveda et al, 2005), los veinte bancos comunitarios de semillas, con su respectivo índice de desarrollo sostenible, y clasificados según su estado del sistema.

Encontrando que los bancos comunitarios de semillas de La Labranza 2, del municipio de Condega, en el departamento de Estelí, en Matagalpa, en el municipio de San Ramón, los bancos comunitarios de Cerro Grande, El Naranjo, La Bailadora y El Horno 2, se encuentran en un estado óptimo de sostenibilidad, atribuyendo esto a factores a la organización, equipamiento y disposición de productores a contribuir con el funcionamiento de banco comunitario.
El banco de la comunidad El Naranjo, del municipio de San Lucas, se clasifica en un estado inestables, las principales razones porque se ubica en este rango es, incumplimiento de pago de semillas, disponibilidad de herramientas, inexistencia de variedades de maíz en el banco, incumplimiento de directivos en las actividades correspondientes al banco, por estas razones se obtuvo el valor mínimo de índice de desarrollo sostenible.

El resto de bancos, se ubican en un estado estable, debido al trabajo que vienen realizando directivos y beneficiarios en la parte organizativa, disponibilidad de herramientas, e insuficientes variedades en los bancos, han prevalecido en las diferentes situaciones que se presentan en el funcionamiento de los bancos comunitarios.

Tabla 2. Agrupación de los bancos comunitarios de semillas según el índice integrado de desarrollo sostenible.

\begin{tabular}{lrr} 
Rangos & Frecuencia & Porcentaje \\
\hline $0-0.20$ & 0 & 0 \\
$0.21-0.40$ & 0 & 0 \\
$0.41-0.60$ & 1 & 5 \\
$0.61-0.80$ & 14 & 70 \\
$0.81-1$ & 5 & 25 \\
\hline
\end{tabular}

La presente tabla refleja que ningún banco se encuentra en estados de colapso y crítico, un banco en estado inestable, catorce en estado estable, y cinco en estado óptimo.

Se observa que los bancos comunitarios, según sus componentes económicos, ambientales y sociales, para alcanzar la sostenibilidad, se encuentran en condiciones favorables con respecto a su funcionamiento. 
Comparar la diversidad de variedades de maíz, frijol y sorgo o millón, entre los bancos comunitarios de semillas criollas (centralizados) con la diversidad de variedades del conjunto de bancos familiares (descentralizados) en las correspondientes comunidades del departamento de Madriz

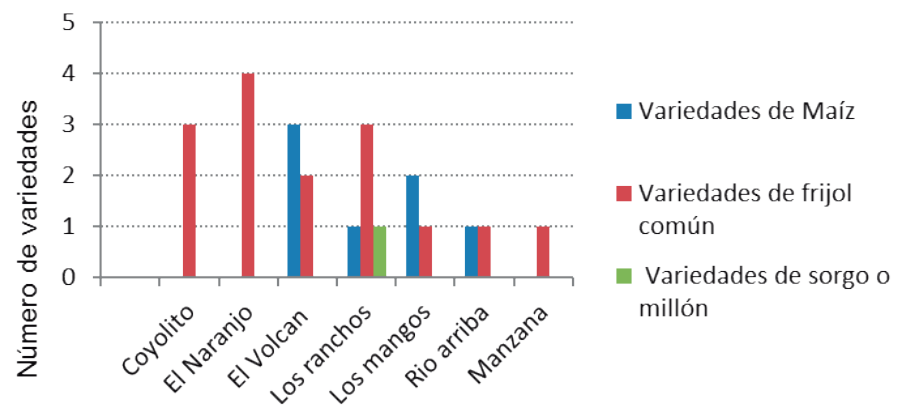

Figura 3.Cantidad de variedades de granos básicos en bancos centralizados en el Departamento de Madriz

El banco que almacena mayor cantidad de variedades de maíz es el banco comunitario del Volcán, seguido por el banco de Los Mangos, Rio Arriba, y Los Ranchos, con dos y una variedad respectivamente, mientras tanto los bancos del el Coyolito, El Naranjo y La Manzana no almacenan ninguna variedad de maíz.

Los bancos comunitarios que almacenan mayor variedades de frijol son El Naranjo, Coyolito y Los Ranchos, con 4 y 3 variedades respectivamente. El banco comunitario del Volcán, Los Mangos, Rio Arriba y La Manzana almacenan 2 y una variedad respectivamente. El banco comunitario de Los Ranchos es donde se encontró una variedad de sorgo.

La figura 10. Refleja las escasas de variedades de granos básicos que se almacenan en los bancos comunitarios de semillas, según los productores, estas razones se presentan por la poca disponibilidad de recipientes para almacenar más variedades de semillas, por falta de organización entre directivos y beneficiarios de semillas para coordinar la introducción de las variedades que más se frecuentan entre productores. La presión de los intermediarios sobre los productores en cosechar semillas con demanda en los mercados nacionales e internacionales es otra limitante. Estas son las principales razones que dificultan la disponibilidad de variedades de semillas en los bancos comunitarios.

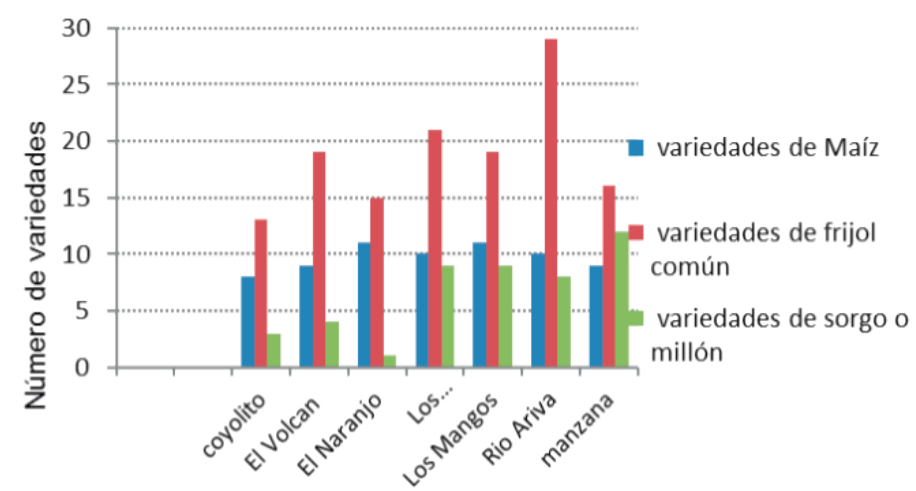

Figura 4. Cantidad de variedades de granos básicos en bancos descentralizados en el Departamento de Madriz.

La figura 4. Representa el número de variedades (maíz, frijoles, sorgo o millón) en 10 bancos familiares o descentralizados de semillas por comunidad, dichos bancos está conformado por socios o beneficiarios de los banco comunitario o centralizados.

En las comunidades del Naranjo y Los Mangos, se encontraron 11 variedades de maíz, en Los Ranchos y Rio Arriba 10 variedades. En variedades de frijoles Rio Arriba cuenta 29 variedades, Los Ranchos con 21 variedades, El Volcán y Los Mangos, ambos con 19 variedades. La Manzana posee 12 variedades de sorgo, Los Ranchos y Los Mangos, ambas con 9 variedades, y rio arriba con 8 variedades, siendo todas estas las cantidades más altas.

El Volcán y La Manzana se encontraron 9 variedades de maíz cada uno, y El Coyolito con 8 variedades. En variedad de frijol, La Manzana y El Naranjo cuentan con 16 y 15 variedades respectivamente, y el Coyolito con 13 variedades, en el sorgo, El Volcán, El Coyolito y El Naranjo, se encontraron 4,3 y 1 variedad respectivamente.

En los bancos descentralizados de semillas criollas y acriolladas del departamento de Madriz se encuentran mayor diversidad de variedades de maíz, frijoles y sorgo, con relación a la cantidad de variedades 
existentes en los bancos centralizados de semillas del mismo departamento.

Datos similares encontraron Pol y Herrera (2005 al 2006), en su investigación "multiplicación de semillas criollas y técnicas campesinas para su almacenamiento, en el municipio de Condega. Trabajaron en 3 comunidades (Labranza 2, Laguna de Santa Rosa y Cooperativa Juanita Vizcaya), tomando la participación en dos comunidades de 10 bancos descentralizados y una de 5 descentralizado, donde encontraron 11,7 y 7 variedades de frijol respectivamente, y de maíz 7,2 y 4 variedades respectivamente.

Ambas investigación concuerdan que la diversidad de variedades de semillas criollas y acriollas se encuentran en los bancos familiares o descentralizados. Estas se han venido conservando tradicionalmente, de generación en generación. La conservación de este patrimonio esta en las manos de las familias campesinas, de igual forma garantizando la seguridad alimentaria de las familias.

\section{Alternativas de funcionamiento de los Bancos Comunitarios de Semillas, mejorando su organización e integrando la diversidad de reservas familiares de cultivos y variedades en cada comunidad, para el avance hacia su sostenibilidad.}

De acuerdo a los grupos focales realizados en cada banco comunitario, en base a las principales fortalezas, amenazas, oportunidades y debilidad expuestas por socios y beneficiarios, encuesta a técnicos de UNAG y según nuestro criterio observado en las visitas a los bancos y reuniones, se proponen las siguientes alternativas para el avance a su sostenibilidad

\section{Aspectos económicos}

Que todos bancos comunitarios de semillas criollas tengan una tasa de interés de recuperación de semillas del $100 \%$, esto con el objetivo de aumentar la cantidad de semillas en beneficio del banco.
Si los prestamistas no logran solventar en tiempo y forma el pago de semillas por alguna razón: (exceso de lluvias, sequillas, plagas, recursos económicos etc.), paguen en el próximo ciclo de cosecha el doble de lo establecido, o con otra especie o variedad, con la intención de mantener el capital del banco.

Para el almacenamiento de la semilla, cada banco debe de contar con el número de silos y otros recipientes necesarios, de acuerdo al número de variedades y a la cantidad de semilla que en este se guarden.

Cada uno de los socios deben comprometerse a devolver una semilla que presente las mismas características a la que se le entrego, para garantizar que se almacenen semillas de buena calidad y evitar que en el banco se presente alguna erosión genética.

En dependencia del aumento de la cantidad de semillas del banco, que se establezcan centros de acopio de granos básicos directamente para la comercialización, en donde se retenga la semilla unos dos meses o más para venderla a un mejor precio, generando un mayor ingreso económico.

En los ciclos productivos, cada directivo del banco asignen una parcela y que la cosecha que obtenga, sea destinada para el banco comunitario.

Acordar con los productores, cuando se obtengan buena producción en la cosecha, devolver más de lo correspondiente.

Crear puestos de ventas donde se promuevan el comercio exclusivo a las variedades criollas y acriolladas de semillas.

Organizar a los productores en cada comunidad y acordar un solo precio para la venta del grano, y tener un precio justo para los intermediarios y consumidores. 


\section{Aspecto ambiental}

Proponer que en todos los banco se implementen técnicas de curado orgánico, para evadir la introducción de sustancias tóxicas en las semillas.

Crear una base de datos de todos los socios y no socios del banco comunitario de semillas en la comunidad, donde refleje todas las especies y sus respectivas variedades de cultivos existentes en sus fincas, y coordinar la introducción de las especies o variedades que más coincidan al banco comunitario.

Asolear el silo un día completo, limpiarlo, luego dejarlo reposar un día completo, para que no se cree humedad en el recipiente.

Que las especies sembradas sean propias de la zona o presenten características adaptables a la misma, para garantizar la producción.

Antes de ser entregada la semilla a los productores, garantizar que está, tenga un porcentaje de germinación que supere el 90\%, para evitar pérdidas en las cosechas por problemas de germinación.

Concientizar y motivar a productores que siembren y conserven las variedades de semillas criollas y acriolladas.

\section{Aspecto social}

Incentivar a los 3 mejores productores pagadores, que cumplan en las fechas establecidas y entreguen semilla de buena calidad de semilla.

Los promotores o directivos de los bancos monitoreen las parcelas de los productores para apoyo técnico.

Anticipadamente los productores deben entregar a la directiva del banco una carta de solicitud, donde refleje la especie, variedad y cantidad de semilla a prestar, luego la directiva analizará cada solicitud y según la disponibilidad de semillas en el banco se dará respuesta a cada solicitud.
En la parte organizativa que cada banco comunitario implementen su reglamento interno, donde plasme los compromisos y deberes que deben asumir los beneficiarios de semillas, un libro de registro, donde especifique: nombredel productor, especie y variedad, cantidad y firma, también la entrega de facturas a los productores, esto para garantizar el control de las semillas.

Autoridades, instituciones, y organizaciones responsables deben establecer estrategias de comercio que permitan incrementar las variedades de granos básicos en los mercados.

Impulsar la divulgación de variedades de semillas criollas y acriolladas por medio de ferias en sus diferentes niveles (municipal, departamental, regional y nacional), donde se resalten la importancia nutritiva de las semillas.

\section{CONCLUSIONES}

Los bancos comunitarios de semillas del departamento de Matagalpa presentan mejor equipamiento, buena organización y mayor cantidad de variedades que los bancos comunitarios de los demás departamentos (Estelí, Madriz y Nueva Segovia).

Según el índice integrado de desarrollo sostenible, la mayoría de los bancos (14) impulsados por el PCAC en el norte de Nicaragua están en estado estable, cinco en estado óptimo y solamente uno en estado inestable, lo que refleja la buena coordinación del programa.

Los bancos familiares o descentralizado del departamento de Madriz presentan mayor diversidad de variedades (maíz, frijoles, sorgo o millón) en comparación a los bancos centralizados o comunitarios del mismo departamento.

Se propusieron alternativas de funcionamiento participativo con técnicos de la UNAG y directivos de los bancos comunitarios de cada departamento en 
estudio con el propósito de contribuir con estrategias para que estos alcancen la sostenibilidad.

\section{RECOMENDACIONES}

A la UNAG-PCaC, y otras instituciones, que trabajan esta temática, sugerimos:

- Mejorar las condiciones de los bancos comunitarios de semillas en cuanto a la infraestructura del local, organización y herramientas de trabajo, para lograr una mayor capacidad de almacenamiento, funcionamiento y resguardo de las semillas.

- Seguir impulsando ferias (municipales, departamentales, regionales y nacionales) entre los bancos comunitarios para preservar nuestro origen ancestral.

- Promover investigaciones sobre semillas criollas y acriolladas como una necesidad de conservar y multiplicar la diversidad de variedades existentes.

- Alos productores, conservar las diversas variedades de semillas criollas y acriolladas que se encuentran en los bancos de semillas familiares.

\section{AGRADECIMIENTO}

Agradecemos primeramente a Dios por ser nuestro amigo incondicional estando siempre a nuestro lado brindándonos su sabiduría y la fortaleza necesaria para seguir siempre adelante. También a nuestros padres, familiares, amigos por sus sabios consejos y apoyo anímico.

Esta investigación fue emprendida gracias a convenio que existe entre la UNAG (Unión Nacional de Agricultores y Ganaderos) - PCaC (Programa de Campesino a campesino) en coordinación con la FAREM- Estelí, con apoyo financiero de SWISSAID (Organismo Suizo no gubernamental de apoyo al desarrollo internacional).

De igual manera se le agradece a la Facultad Regional Multidisciplinaria de Estelí por haber contribuido en nuestra formación profesional y haber incluido en nosotros valores éticos y morales que nos fueron muy útiles en la culminación de nuestra carrera .

A la UNAG, Andreu Pol, Jorge Irán, Técnicos de la UNAG de: Estelí, Madriz, Nueva Segovia y Matagalpa, por habernos brindado información necesaria para la redacción de nuestro documento y ayudarnos a establecer comunicación con cada uno de los productores en cada departamento.

A todos los docentes de la facultad por habernos trasmitidos sus conocimientos en cada una de las asignaturas impartidas durante los 5 años de la carrera que nos servirán de una u otra forma en aras de contribuir en la solución de problemas ambientales de nuestro País.

A nuestro tutor Lic. Oscar Rafael Lanuza, y asesores MSc. Alejandrina Herrera y MSc. Kenny López Benavides por habernos dado el tiempo necesario en correcciones, aportes y sugerencias científico metodológico para mejorar nuestro trabajo.

Agradecemos a todos los productores de cada uno de los departamentos donde realizamos el estudio por habernos brindado su atención y haber contribuido a brindar información importante para darle salida a nuestros objetivos.

\section{BIBLIOGRAFÍA}

Altieri, M. (1985). Agroecología: Bases científicas de la agricultura alternativa. Chile: Cetal.

Binder, U. (1997). Manual de leguminosas de Nicaragua Tomo 1. Estelí,Nicaragua: PASOLAC. Binder, U. (1997). Manual de leguminosas de Nicaragua Tomo 2. Estelí,Nicaragua: PASOLAC. CURN-Estelí, PCaC. (2006). Multiplicacion de Semillas Criollas y Tecnicas Campesinas para su Almacenamiento en el Municipio de Condega, pp.74.

Barquero, J. I. (2000). Geografía Dinámica de Nicaragua. Mangua: HISPAMER. 
Fulgani Guaharay, M. c. (2012). Sistematizacion de experiencias y aprendizajes de campo sobre bancos comunitarios de semilla por organismos nicaraguences aliados en la plataforma zona alta de matagalpa. Bancos comuniarios de semilla, pp. 46.

Gispert, C. (2008). Atlas Geográfico de Nicaragua. España: Oceano.

Laurent Levard, Y. M. (1999). La problematica técnica y la intervención de los organismos que trabajan en el fenómeno tecnológico en el trópico de Nicaragua. Estudios Centroamericanos, 18.

Ley General del Medio Ambiente y los Recursos Naturales, Managua, 1996.

INTA, (2002). Guia Tecnológica 1. Generalidades sobre los Granos Básicos, pag.5-6.

IPCC. (2002). Cambio climático y biodiversidad. Grupo intergubernamental de expertos sobre el cambio climático, pag. 82.

José Egea Fernández, J. G. (2013). Estado de los recurso fitogéneticos desde la perpectiva de las redes de familia. Agroecología 7 (2), 63.

MARENA. (2004). Atlas Escolar. Managua: EDITARTE

MAGFOR. (2012). Informe estadístico de la producción agropecuaria . Managua.

Nicaragua, P. (2011). Coleccion familias trabajando juntas por la seguridad alimentaria. Guia para organizar los bancos comunitarios de semilla , 18 pág.

Pol, A., Vásquez, J. (2007). Curado Orgánico de Semillas Criollas, Cartilla 1 Colección Experiencias sobre el Rescate de Semillas Criollas, PCaCUNAG y SWISSAID, Managua, pp. 48.

Pol, A., Vásquez J. (2009) Red Nacional de Bancos Comunitarios de Semillas criollas, Cartilla 2 Colección Experiencias sobre el Rescate de Semillas Criollas, PCaC-UNAG y SWISSAID, Managua, pp.46.
Pol, A., Vásquez J. (2011) Comercialización y Ferias de Intercambio de Semillas y de Productos Criollos, Cartilla 3 Colección Experiencias sobre el Rescate de Semillas Criollas, PCaC-UNAG y SWISSAID, Managua pp.44.

Pol, A., Vásquez J. (2012) Mejoramiento Campesino de Semillas Criollos, su Multiplicación y Diversificación de Variedades Criollas Cartilla 4 Colección Experiencias sobre el Rescate de Semillas Criollas, PCaC-UNAG y SWISSAID, Managua, pp. 32.

Pavon, F. d. (2009). Evauación participativa de indicadores de desarrollo sostenible en sistemas de producción de pequeña escala en el municipio de la Sabana, Madriz. Estelí: Universidad Autonoma de Barcelona.

Sergio Sepúlveda, H. C. (2005). Metodología para estimar el nivel de desarrollo sostenible de los territorios rurales. ( El biograma), pp. 109.

UNAG-PCAC. (2004). La Semillas Criollas,nuestra Herencia, nuestra Tradición, nuestro Alimento. Managua.

UICN, (2008). Acción ante el Cambio Climático. Cumbre de la Sociedad Civil por el Cambio Climático, pp.16.

INTA, (2002). Guia Tecnológica 1. Generalidades sobre los Granos Básicos, Paginas 5-6.

Sampieri, (1997). Metodologia de la investigacion. Mexico: MCGRAW - HILL.

SIMAS, (2012). Sistematizacion de experiencias y aprendizajes del campo sobre los bancos comunitarios de semilla por organismos nicaraguences aliados en la plataforma zona alta de matagalpa. (46).

Zamora C., A. (2011). Guia para la Organización de los Bancos Comunitarios de Semillas. De grano en grano se llena el silo, pp.12. 